\title{
Non-destructive State Detection and Spectroscopy of Single Molecules
}

\author{
Mudit Sinhal§ ${ }^{\S}$ Ziv Meir, and Stefan Willitsch* \\ §SCS-Metrohm award for the best oral presentation in Physical Chemistry
}

\begin{abstract}
We review our recent experimental results on the non-destructive quantum-state detection and spectroscopy of single trapped molecules. At the heart of our scheme, a single atomic ion is used to probe the state of a single molecular ion without destroying the molecule or even perturbing its quantum state. This method opens up perspectives for new research directions in precision spectroscopy, for the development of new frequency standards, for tests of fundamental physical concepts and for the precise study of chemical reactions and molecular collisions with full control over the molecular quantum state.
\end{abstract}

Keywords: Cold molecular ions · Ion traps $\cdot \mathrm{N}_{2}^{+}$. Precision spectroscopy $\cdot$ Quantum-logic spectroscopy

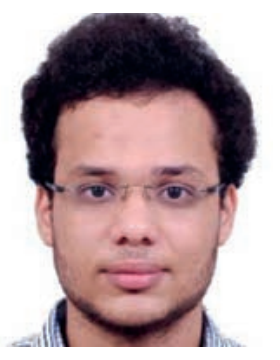

Mudit Sinhal received his integrated MSc (2016) in Physics at the Indian Institute of Technology (Kharagpur, India). During his studies, he pursued internships in the groups of Prof. Dr. Kenneth Brown at Georgia Institute of Technology (Georgia, USA) and Prof. Dr. Jonathan Home at ETH Zurich (Zurich, Switzerland) focusing on trapped ions. Since 2016, he is a PhD student in the group of Prof. Dr. Stefan Willitsch at the University of Basel (Basel, Switzerland). His research interests focus on development of novel quantum technologies like high-spectralpurity electromagnetic sources and quantum-state manipulation techniques for precision measurements on single atoms and molecules.

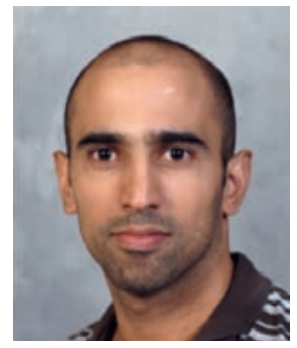

Ziv Meir is currently a postdoctoral fellow in the University of Basel in the group of Prof. Dr. Stefan Willitsch. He graduated in both Physics and Electrical Engineering from Tel-Aviv University in 2009 and received his $\mathrm{PhD}$ from the Weizmann Institute of Science in 2016. In his PhD thesis, he studied the dynamics of collisions between ultracold atoms and ions in overlapping traps. In Basel, he is focused on applying quantum protocols for studying molecular ions.

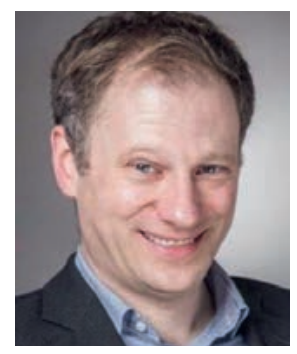

Stefan Willitsch graduated in natural sciences from ETH Zurich in 2000 and received his PhD from ETH in 2004 with a dissertation on high-resolution photoelectron spectroscopy. From 2004-07, he was a Junior Research Fellow at the University of Oxford. He was appointed lecturer in physical chemistry at University College London in 2007 and joined the Department of Chemistry at the University of Basel in 2008. His research in- terests focus on translationally cold molecules and ions and their applications in spectroscopy, chemical dynamics and quantum technologies.

\section{Introduction}

The ability to trap, cool and manipulate atoms, molecules and ions has recently led to impressive advancements in both chemistry and physics. ${ }^{[1-3]}$ These technologies have enabled experiments with long interrogation times under precisely controlled conditions paving the way for applications such as extremely precise spectroscopic measurements ${ }^{[4-6]}$ thus facilitating, e.g., searches for new physics beyond the standard model ${ }^{[7,8]}$ and accurate tests of high-level theoretical calculations of atomic and molecular energy-level structures. ${ }^{[9,10]}$ These technologies have also enabled precise studies of chemical reaction and collision dynamics ${ }^{[1-13]}$ and of state- and energy-controlled reactions under cold and ultracold conditions. ${ }^{[14-18]}$

At the core of many of these experiments are atoms or atomic ions which can be Doppler laser cooled on closed optical cycling transitions. These systems form the basis of the most precise frequency standards, ${ }^{[5,19,20]}$ they have been used to perform quantum simulations and quantum computations ${ }^{[21,22]}$ and they are also employed in new experiments to study chemistry at extremely low temperatures. ${ }^{[23-25]}$ By contrast, most molecules lack closedcycling electronic transitions owing to their complex energy-level structure. Thus, the non-destructive techniques for internal-state preparation, laser cooling, state detection and state manipulation which are routinely used for specific atoms ${ }^{[26]}$ cannot, in general, be directly adapted to molecular systems.

In order to overcome these shortcomings, we have developed novel technologies for the quantum-manipulation of single molecules. ${ }^{[27-29]}$ We have adapted quantum-logic techniques ${ }^{[30]}$ in which a single molecular ion is simultaneously trapped with a single laser-cooled atomic ion in a radiofrequency (rf) quadrupole ion trap. ${ }^{[29]}$ The cooling and state detection of the molecular ion is mediated via the atomic ion through their mutual Coulomb interaction which is also employed in related quantum-logic experiments with both atomic ${ }^{[31]}$ and molecular ions. ${ }^{[32-35]}$ In the 
present experiments, we use the ${ }^{14} \mathrm{~N}_{2}^{+}$molecular ion and the ${ }^{40} \mathrm{Ca}^{+}$ atomic ion as prototypical examples. However, the methods presented here are general and applicable to a broad range of systems.

$\mathrm{N}_{2}^{+}$is of particular interest for the present experiments as it is a homonuclear diatomic ion with no permanent electric-dipole moment. Therefore, all rovibrational transitions within the same electronic state are forbidden within the electric-dipole approximation. ${ }^{[36]}$ However, due to higher order terms in the multipole expansion of the interaction of matter with radiation, weak electric-quadrupole, magnetic-dipole and higher-order rovibrational transitions are allowed. ${ }^{[36-38]}$ Such weak transitions exhibit extremely narrow natural linewidths and consequently very long state lifetimes. These properties render $\mathrm{N}_{2}^{+}$an ideal test-bed for precision spectroscopic studies, ${ }^{[37,38]}$ for chemical reactions and collision studies in specific internal states, ${ }^{[16,39-41]}$ for the realization of mid-IR frequency standards and molecular clocks, ${ }^{37,42]}$ for tests of possible temporal variations in the electron-to-proton mass ratio ${ }^{[43]}$ and for the implementation of molecular qubits used in quantum information and computation applications..$^{[37]}$

\section{Non-destructive State Detection of Single Trapped Molecular Ions}

Efficient methods for the determination of atomic and molecular quantum states (and their changes) are at the heart of sensitive experiments in both quantum logic and precision spectroscopy. In many atomic systems, the closed optical-cycling transitions that are used for laser cooling are also employed for non-destructive state readout. [31] By contrast, molecular ions without such transitions have in the past often been read out using destructive action-spectroscopic methods ${ }^{[44]}$ such as state-dependent chemical reactions ${ }^{[42,45]}$ and resonance-enhanced multi-photon dissociation (REMPD). ${ }^{[4]}$ While these schemes enable state detection on the single-particle level, the molecular state and even the molecule itself is destroyed in each measurement. Consequently, this necessitates large ensembles of molecules to improve the measurement sensitivity ${ }^{[23,24]}$ as the molecular ions have to be replenished after each measurement which greatly reduces the duty cycles of experiments. The averaging over ensemble properties and the low duty cycle severely limit the achievable accuracy, precision and signal-to-noise ratio of spectroscopic measurements. Thus, the development of non-destructive internal-state detection techniques has been a long-standing problem in the field of molecular ions.

We have recently demonstrated such a technique for single molecular ions.[27] The principle of the method is illustrated in Fig. 1. Our approach relied on exciting motion in a $\mathrm{Ca}^{+}-\mathrm{N}_{2}^{+}$twoion string by a state-dependent optical-dipole force (ODF). The ODF is generated by the interaction of off-resonant light with a strong spectroscopic resonance in the molecular ion. Due to the dispersive nature of the coupling, no photon is scattered off the molecule and the internal state of the $\mathrm{N}_{2}^{+}$ion is preserved. Thus, repeated measurements on the same molecule can be carried out without destroying it. The present experiments focused on $\mathrm{N}_{2}^{+}$ions in the $X^{2} \Sigma_{g}^{+}(v=0, N=0)$ rovibronic ground state, where $v$ and $N$ denote the vibrational and rotational quantum numbers, respectively.

In a first step, Doppler laser cooling was employed to cool the external motion of the ions. ${ }^{[23,26]}$ This technique relies on the continuous scattering of photons on closed-cycling transitions, here the $(4 s)^{2} S_{1 / 2} \leftrightarrow(4 p)^{2} P_{1 / 2} \leftrightarrow(3 d)^{2} D_{3 / 2}$ set of resonances in $\mathrm{Ca}^{+}$. By tuning the frequency of the cooling laser slightly to the red of the transition, kinetic energy is removed from the atomic ion by the repeated absorption of photons and cooling to temperatures in the $\mathrm{mK}$ range can be achieved. Doppler cooling reduces the Doppler shifts of the spectral lines under investigation and leads to the spatial localization of the ions in the trap forming ordered structures termed 'Coulomb crystals'. ${ }^{[23]}$ Since the $\mathrm{N}_{2}^{+}$ions in the

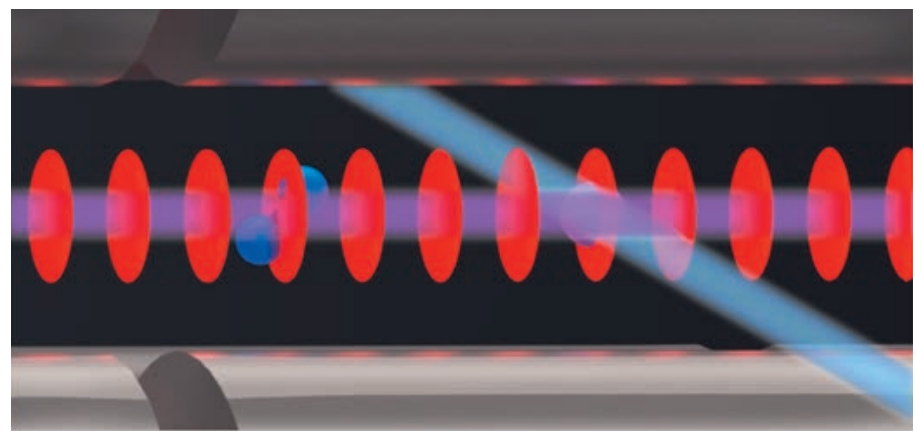

Fig. 1. Illustration of the present method for the non-destructive quantum-state detection of single ${ }^{14} \mathrm{~N}_{2}^{+}$molecular ions with single ${ }^{40} \mathrm{Ca}^{+}$ atomic ions. Both species (molecule in blue and atom in purple) were simultaneously trapped in an ion trap (grey rods). The $\mathrm{N}_{2}^{+}$molecular ion was sympathetically cooled by the laser-cooled $\mathrm{Ca}^{+}$ion $\left(\mathrm{Ca}^{+}\right.$coolinglaser beam in blue). A one-dimensional optical lattice (red) was used to apply a state-dependent optical-dipole force on the molecular ion and excite motion of the two-ion crystal depending on the internal state of $\mathrm{N}_{2}^{+}$. Another laser beam (purple) was used to detect the motional excitation of the two-ion crystal by Rabi spectroscopy on $\mathrm{Ca}^{+}$. Dimensions not to scale.

trap could not be directly laser cooled due to absence of closedcycling transitions, their motion was cooled sympathetically by their long-range Coulomb interactions with co-trapped lasercooled $\mathrm{Ca}^{+}$ions. ${ }^{[29]}$

In order to further cool the two-ion string, a subsequent cooling step known as resolved-sideband cooling was used.[26] In this regime, the quantized motion of the particles in the harmonic potential of the ion trap is directly addressed. The quantized modes of motion of the ions appear as sidebands in the spectrum of narrow spectroscopic transitions of the trapped species. Here, one of the modes of the $\mathrm{Ca}^{+}-\mathrm{N}_{2}^{+}$string was cooled sympathetically to its quantum-mechanical ground state by driving its sidebands on the $(4 s)^{2} S_{1 / 2} \rightarrow(3 d)^{2} D_{5 / 2}$ electronic transition in $\mathrm{Ca}^{+} .{ }^{[29]}$

The ODF for state detection in our experiments ${ }^{[27-29]}$ was implemented by two counter-propagating laser beams which formed a one-dimensional optical lattice. The strength of the ODF is enhanced when the optical frequency of the lattice is near a strong spectroscopic resonance in the molecule. Consequently, the laser frequency of the lattice, $f_{\text {lattice }}$, was set close to the $A^{2} \Pi_{u}(v=2) \leftarrow X^{2} \Sigma_{g}^{+}(v=0) R_{11}(1 / 2)$ spectroscopic transition ${ }^{[46]}$ originating from the rovibronic ground state of $\mathrm{N}_{2}^{+}$. In addition, the amplitude of the ODF was modulated in resonance with the motional frequency of the ion crystal. This generated a resonant drive which resulted in a significant motional excitation of the initially ground-state cooled mode of the $\mathrm{Ca}^{+}-\mathrm{N}_{2}^{+}$crystal conditional on whether the $\mathrm{N}_{2}^{+}$ion was in its rovibronic ground state. An $\mathrm{N}_{2}^{+}$ion in the rovibronic ground state experienced a strong ODF which resulted in a strongly excited motion. By contrast, molecules in an excited rotational or vibrational state experienced a much weaker ODF due to the lattice laser being farther detuned from any possible spectroscopic transition originating from these states. The resulting motional excitation was consequently much smaller.

The state-dependent ODF thus mapped the information of the internal state of the molecule onto the external motion of the $\mathrm{Ca}^{+}-\mathrm{N}_{2}^{+}$crystal. The excited motion was subsequently detected by Rabi sideband spectroscopy on the $\mathrm{Ca}^{+}$ion to reveal the state of the molecule. Further details about the experimental implementation of the technique can be found in refs. [27-29].

\section{Exemplary Results}

A demonstration of the present state detection technique is shown in Fig. 2.[27] The duration of a narrow $729 \mathrm{~nm}$ laser pulse, $t_{729}$, in resonance with a motional sideband on the $\mathrm{Ca}^{+}(4 s)^{2} S_{1 / 2} \rightarrow(3 d)^{2} D_{5 / 2}$ transition was scanned in order to induce 

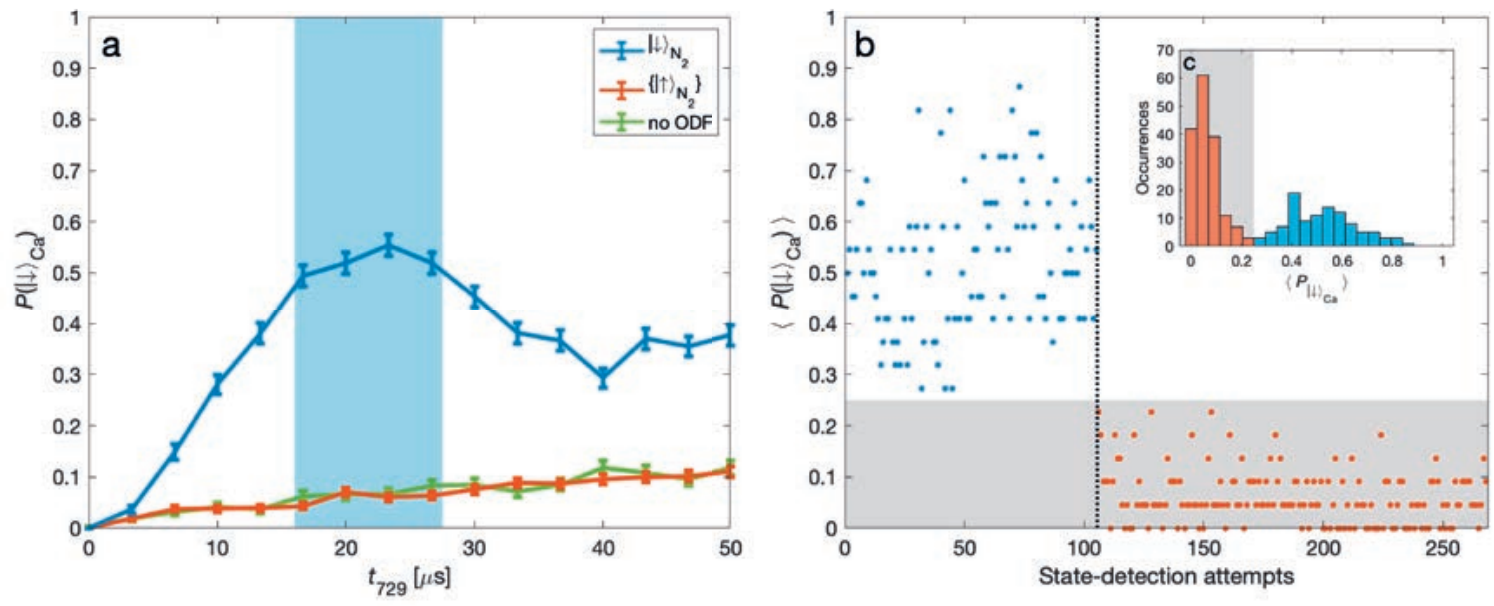

Fig. 2. Non-destructive state detection of single $\mathrm{N}_{2}^{+}$ions. ${ }^{[27]}$ a) Rabi-oscillations on a motional sideband of the $(4 s)^{2} S_{1 / 2} \rightarrow(3 d)^{2} D_{5 / 2}$ transition in $\mathrm{Ca}^{+}$for $\mathrm{N}_{2}^{+}$ in the ground rovibronic state (blue trace) and in a rotationally excited state (red trace). The green trace corresponds to a background measurement of the Rabi oscillation signal without the state-dependent optical dipole force applied. The light-blue region indicates the Rabi sideband-pulse durations for which maximum state-detection contrast was achieved. b) Time trace of state-detection attempts. A single state-detection data point is composed of an average of 22 consecutive sideband-measurements for pulse durations indicated by the light-blue area in (a). The dashed black line indicates the occurrence of a quantum jump of the molecule out of its ground state. c) Histogram of state-detection attempts. The grey area separates between ground- (blue bars) and excited- (red bars) state-detection assignments (same as (b)). From ref. [27]. Reprinted with permission from AAAS.

coherent Rabi oscillations. The population underwent periodic oscillations between the two states of the $\mathrm{Ca}^{+}$ion due to stimulated absorption and emission of photons induced by the $729 \mathrm{~nm}$ laser. With $\mathrm{N}_{2}^{+}$in its rovibronic ground state, strong Rabi oscillations (blue trace) were observed due to excitation of motion by the ODF, while with the molecule in an excited rotational state, almost no oscillations were observed (orange trace).

Each of the traces shown in Fig. 2 consisted of an average of thousands of measurements performed on the same molecular ion which was possible due to the non-destructive nature of the detection scheme. While these thousands of measurements unambiguously differentiate the molecular ground state from all excited states, much fewer measurements are in fact needed to achieve a high-fidelity state detection. Fig. 2b depicts the molecular state as a function of state detection attempts where each attempt consisted of only 22 Rabi pulses for which the pulse time was set within the region of maximum contrast indicated by the light-blue area in Fig. 2a. In this case, a state-detection attempt took only few $100 \mathrm{~ms}$ which allowed us to track the molecular state with a high time resolution. In Fig. 2b, a time trace of the molecular state is given. For the measurements recorded at the beginning of the experiment (blue dots), the molecule was determined to be in the rovibronic ground state 105 times with zero false detection events. At this point, the molecule abruptly changed its state and was detected not to be in the rovibronic ground state 163 times with zero false detection attempts (orange data points). It is most likely that the molecule underwent a quantum jump out of the rovibronic ground state due to a collision with a background gas molecule. From this measurement, an experimental fidelity of $>99 \%$ for the faithful detection of the rovibronic ground state was determined.

As an application of this state-detection technique, a non-invasive spectroscopic measurement on the $A^{2} \Pi_{u}(v=2) \leftarrow X^{2} \Sigma_{g}^{+}(v=0) R_{11}(1 / 2)$ transition of $\mathrm{N}_{2}^{+}$was performed exploiting the fact that the excited motion of the two-ion crystal is proportional to the ac-Stark shift experienced by the molecule. ${ }^{[27,29]}$ The ac-Stark shift, which gives rise to the ODF, scales as $\sim 1 / \Delta$ where $\Delta=f_{\text {lattice }}-f_{0}$ is the frequency detuning of the lattice beams from the $R_{11}(1 / 2)$ resonance, $f_{0}$. Rabi spectroscopy was performed at different detunings of the lattice laser from the expected position of the resonance. ${ }^{[46-48]}$ The magnitude of the ac-Stark shift was extracted from a fit to the observed Rabi oscillations.
Fig. 3 depicts such a 'force spectrum' of the molecular transition. The obtained spectrum was fitted to a $\sim 1 /\left|f-f_{0}\right|$ ac-Stark shift profile in order to determine the line center. Our measurement of $f_{0}=380.7011(2) \mathrm{THz}$ agreed well with the previous experiments $^{[46-48]}$ in the range $f_{0}=380.7007(3) \mathrm{THz}$ which were performed with traditional spectroscopic techniques using ensembles of molecules. Furthermore, the vibronic Einstein- $A$ coefficient of this transition, $A_{\text {vibronic }}$, was determined from the measurements. For each measured ac-Stark shift at a particular lattice-laser detuning, a corresponding $A_{\text {vibrinic }}$ was extracted and a mean value of $A_{\text {vibronic }}=3.98(11) \times 10^{4} \mathrm{~s}^{-1}$ was found in good agreement with previous results ${ }^{[49-51]}$ in the range $A_{\text {vibronic }}=3.87(14) \times 10^{4} \mathrm{~s}^{-1}$.

\section{Conclusion and Outlook}

We have demonstrated a novel non-destructive state-detection technique for single molecular ions in a trap. The method is based on a state-dependent coherent excitation of the shared motion of simultaneously trapped single atomic and molecular ions using an optical dipole force and the subsequent probing of the amplitude of motion by spectroscopy on the atomic ion. The detection fidelities achieved exceed $99 \%$ and are currently limited by experimental imperfections such as collisions with background gas molecules in the vacuum system. On the basis of the present state-detection scheme, a non-invasive 'force-spectroscopic' technique for measuring line positions and transition strengths was demonstrated. While we used $\mathrm{N}_{2}^{+}$as a prototypical example in our experiments, the present techniques are general and can be adapted for a wide range of diatomic, polyatomic, polar and apolar molecules.

The present scheme enables experiments with trapped molecules with duty cycles several orders of magnitudes higher than previously achievable with destructive techniques. ${ }^{[4,42]}$ At the same time, the need for interrogating large ensembles of molecules to obtain a sufficient signal-to-noise ratio is eliminated. Thus, the present method is expected to yield vast improvements in the precision of spectroscopic experiments on molecules. This increased sensitivity of measurements and high degree of control are a prerequisite for the application of single molecules as frequency standards and as quantum bits. ${ }^{[37]}$ It also opens perspectives for studies of collisions and chemical reaction between ions and neutrals with precise state control on the single molecule level. 

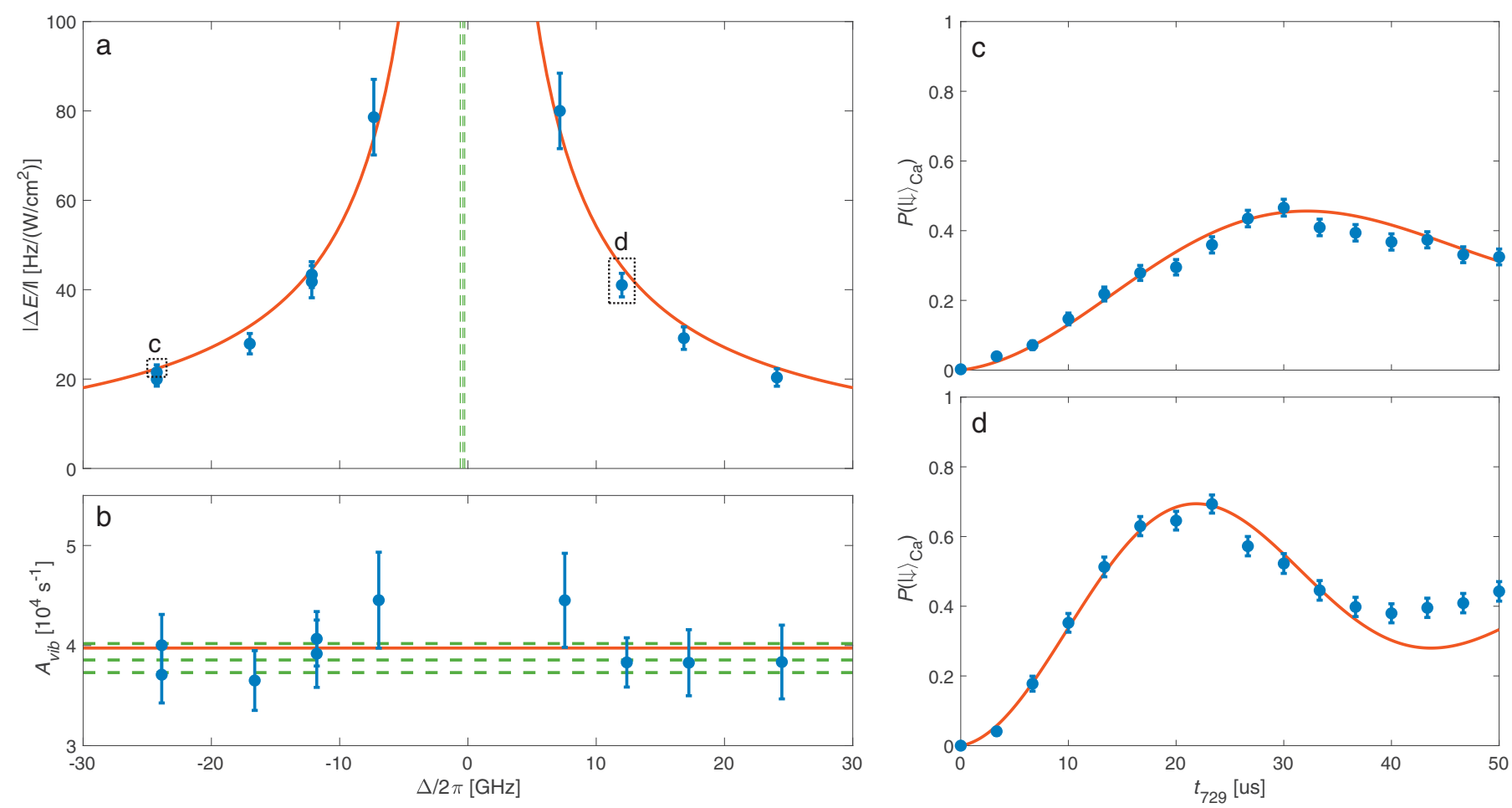

Fig. 3. Non-destructive force spectroscopy on a single $\mathrm{N}_{2}^{+}$molecular ion ${ }^{[2]}$ with the example of the $A^{2} \Pi_{u}(v=2) \leftarrow X^{2} \Sigma_{g}^{+}(v=0) R_{11}(1 / 2)$ transition. a) The blue data points represent the amplitude of the ac-Stark shift, $|\Delta E|$, which gives rise to the ODF experienced by the $\mathrm{N}_{2}^{+}$ion as a function of the frequency detuning from resonance, $\Delta / 2 \pi$. The data were normalised by the lattice-laser intensity, $I$, and were extracted from fits to sideband Rabi-oscillation signals (insets (c), (d)). The red line is a fit to the experimental data used to determine the line center. The green dashed lines correspond to line positions reported in the literature. ${ }^{[46-48]}(\mathrm{b})$ The blue data points represent Einstein-A coefficients of the $A^{2} \Pi_{u}(v=2) \rightarrow X^{2} \Sigma_{g}^{+}(v=0)$ vibronic transition in $\mathrm{N}_{2}^{+}$extracted from the measurements in (a). The red line is the mean of all measurements. Different literature values ${ }^{[49-51]}$ are indicated by the dashed green lines. c), d) Two examples of observed Rabi oscillation signals (blue points) for the $\mathrm{N}_{2}^{+}$ion in the ground state for lattice laser detunings of $\sim-35 \mathrm{GHz}$ and $\sim+15 \mathrm{GHz}$ respectively. The red line is a fit to determine the ac-Stark shift experienced by the molecule at the relevant lattice detunings. From ref. [27]. Reprinted with permission from AAAS.

\section{Acknowledgements}

We thank A. Johnson, P. Knöpfel, G. Martin, and G. Holderied for technical support. We also thank Dr. K. Najafian and G. Hegi for their contributions to the developments reviewed here. M.S. thanks the Swiss Chemical Society and Metrohm for the award of the prize for best oral presentation in the field of Physical Chemistry at the SCS Fall Meeting 2020. We acknowledge financial support from the Swiss National Science Foundation as part of the National Centre of Competence in Research, Quantum Science and Technology (NCCR-QSIT) and grant nr. CRSII5 183579 as well as the University of Basel.

Received: Jamuary 29, 2021

[1] H. J. Metcalf, P. van der Straten, 'Laser Cooling and Trapping' Springer, 1999.

[2] R. V. Krems, W. C. Stwalley, B. Friedrich, 'Cold Molecules: Theory, Experiment, Applications' CRC Press, 2009.

[3] F. G. Major, V. N. Gheorghe, G. Werth, 'Charged Particle Traps' Springer, 2005.

[4] S. Patra, M. Germann, J.-Ph. Karr, M. Haidar, L. Hilico, V. I. Korobov, F. M. J. Cozijn, K. S. E. Eikema, W. Ubachs, J. C. J. Koelmeij, Science 2020, 369, 1238, https://doi.org/10.1126/science.aba0453

[5] S. Brewer, J.-S. Chen, A. M. Hankin, E. R. Clements, C. W. Chou, D. J. Wineland, D. B. Hume, D. R. Leibrandt, Phys. Rev. Lett. 2019, 123, 033201, https://doi.org/10.1103/PhysRevLett.123.033201

[6] S. Dörscher, N. Huntemann, R. Schwarz, R. Lange, E. Benkler, B. Lipphardt, U. Stzerr, E. Peik, C. Lisdat, Metrologia 2020, 58, 015005, https://doi.org/10.1088/1681-7575/abc86f

[7] M. S. Safronova, D. Budker, D. DeMille, D. F. Jackson Kimball, A. Derevianko, C. W. Clark, Rev. Mod. Phys. 2018, 90, 025008, https://doi.org/10.1103/RevModPhys.90.025008

[8] D. DeMille, J. M. Doyle, A. O. Sushkov, Science 2017, 357, 990, https://doi.org/10.1126/science.aal3003
[9] M. Safronova, U. Safronova, Phys. Rev. A 2011, 83, 012503, https://doi.org/10.1103/PhysRevA.83.012503

[10] Z. Meir, M. Sinhal, M. S. Safronova, S. Willitsch, Phys. Rev. A 2020, 101, 012509, https://doi.org/10.1103/PhysRevA.101.012509

[11] Y.-P. Chang, K. Dlugoecki, J. Küpper, D. Rösch, D. Wild, S. Willitsch, Science 2013, 342, 98, https://doi.org/10.1126/science.1242271

[12] A. Kilaj, H. Gao, D. Rösch, U. Rivero, J. Küpper, S. Willitsch, Nat. Commun. 2018, 9, 1, https://doi.org/10.1038/s41467-018-04483-3

[13] S. N. Vogels, J. Onvlee, S. Chefdeville, A. van der Avoird, G. C. Groenenboom, S. Y. T. van de Meerakker, Science 2015, 350, 787, https://doi.org/10.1126/science.aad2356

[14] A. B. Henson, S. Gersten, Y. Shagam, J. Narevicius, E. Narevicius, Science 2012, 338, 234, https://doi.org/10.1126/science.1229141

[15] S. D. S. Gordon, J. J. Omiste, J. Zou, S. Tanteri, P. Brumer, A. Osterwalder Nat. Chem. 2018, 10, 1190, https://doi.org/10.1038/s41557-018-0152-2

[16] A. D. Dörfler, P. Eberle, D. Koner, M. Tomza, M. Meuwly, S. Willitsch, Nat. Commun. 2019, 10, 5429, https://doi.org/10.1038/s41467-019-13218-x y

[17] T. Sikorsky, Z. Meir, R. Ben-shlomi, N. Akerman, R. Ozeri, Nat. Comm. 2018, 9, 920, https://doi.org/10.1038/s41467-018-03373-y

[18] P. Allmendinger, J. Deiglmayr, K. Höveler, O. Schullian, F. Merkt, J. Chem. Phys 2016, 145, 244316, https://doi.org/10.1063/1.4972130

[19] B. J. Bloom, T. L. Nicholson, J. R. Williams, S. L. Campbell, M. Bishof, X. Zhang, W. Zhang, S. L. Bromley, J. Ye, Nature 2014, 506, 71, https://doi.org/10.1038/nature12941

[20] N. Huntemann, C. Sanner, B. Lipphardt, C. Tamm, E. Peik, Phys. Rev. Lett. 2016, 116, 063001, https://doi.org/10.1103/PhysRevLett.116.063001

[21] R. Blatt, D. Wineland, Nature 2008, 453, 1008, https://doi.org/10.1038/nature07125

[22] K. Wright, K. M. Beck, S. Debnath, J. M. Amini, Y. Nam, N. Grzesiak, J.-S. Chen, N. C. Pisenti, M. Chmielewski, C. Collins, K. M. Hudek, J. Mizrahi, J. D. Wong-Campos, S. Allen, J. Apisdorf, P. Solomon, M. Williams, A. M. Ducore, A. Blinov, S. M. Kreikemeier, V. Chaplin, M. Keesan, C. Monroe, J. Kim, Nat. Commun. 2019, 10, 1, https://doi.org/10.1038/s41467-019-13534-2

[23] S. Willitsch, Int. Rev. Phys. Chem. 2012, 31, 175, https://doi.org/10.1080/0144235X.2012.667221

[24] S. Willitsch, Adv. Chem. Phys. 2017, 162, 307, https://doi.org/10.1002/9781119324560.ch5 
[25] O. Dulieu, A. Osterwalder, 'Cold Chemistry: Molecular scattering and reactivity near absolute zero' Royal Society of Chemistry, 2017.

[26] D. Leibfried, R. Blatt, C. Monroe, D. Wineland, Rev. Mod. Phys. 2003, 75 281, https://doi.org/10.1103/RevModPhys.75.281

[27] M. Sinhal, Z. Meir, K. Najafian, G. Hegi, S. Willitsch, Science 2020, 367 1213, https://doi.org/10.1126/science.aaz9837

[28] K. Najafian, Z. Meir, M. Sinhal, S. Willitsch, Nat. Commun. 2020, 11, 4470, https://doi.org/10.1038/s41467-020-18170-9

[29] Z. Meir, G. Hegi, K. Najafian, M. Sinhal, S. Willitsch, Faraday Discuss. 2019, 217, 561, https://doi.org/10.1039/C8FD00195B

[30] P. O. Schmidt, T. Rosenband, C. Langer, W. M. Itano, J. C. Bergquist, D. J. Wineland, Science 2005, 309, 749, https://doi.org/10.1126/science.1114375

[31] H. Häffner, C. F. Roos, R. Blatt, Phys. Rep. 2008, 469, 155, https://doi.org/10.1016/j.physrep.2008.09.003

[32] C. Chou, A. L. Collopy, C. Kurz, Y. Lin, M. E. Harding, P. N. Plessow, T. Fortier, S. Diddams, D. Liebfried, D. R. Leibrandt, Science 2020, 367, 1458, https://doi.org/10.1126/science.aba3628

[33] C. W. Chou, C. Kurz, D. B. Hume, P. N. Plessow, D. R. Leibrandt, D. Leibfried, Nature 2017, 545, 203, https://doi.org/10.1038/nature22338

[34] F. Wolf, Y. Wan, J. C. Heip, F. Gebert, C. Shi, P. O. Schmidt, Nature 2016, 530, 457, https://doi.org/10.1038/nature16513

[35] Y. Lin, D. R. Leibrandt, D. Leibfried, C.-W. Chou, Nature 2020, 581, 273, https://doi.org/10.1038/s41586-020-2257-1

[36] M. Germann, S. Willitsch, Mol. Phys. 2016, 114, 769, https://doi.org/10.1080/00268976.2015.1118568

[37] K. Najafian, Z. Meir, S. Willitsch, Phys. Chem. Chem. Phys. 2020, 22, 23083, https://doi.org/10.1039/D0CP03906C

[38] M. Kajita, Phys. Rev. A 2015, 92, 043423, https://doi.org/10.1103/PhysRevA.92.043423

[39] A. Dörfler, E. Yurtsever, P. Villareal, T. Gonzalez-Lezana, F. A. Gianturco, S. Willitsch, Phys. Rev. A 2020, 101, 012706, https://doi.org/10.1103/PhysRevA.101.012706

[40] F. A. Gianturco, A. D. Dörfler, S. Willitsch, E. Yurtsever, T. GonzlezLezana, P. Villareal, Phys. Chem. Chem. Phys. 2019, 21, 8342, https://doi.org/10.1039/C8CP06761A
[41] X. Tong, T. Nagy, J. Y. Reyes, M. Germann, M. Meuwly, S. Willitsch, Chem. Phys. Lett. 2012, 547, 1, https://doi.org/10.1016/j.cplett.2012.06.042

[42] M. Germann, X. Tong, S. Willitsch, Nat. Phys. 2014, 10, 820, https://doi.org/10.1038/nphys3085

[43] M. Kajita, G. Gopakumar, M. Abe, M. Hada, M. Keller, Phys. Rev. A 2014 89, 032509, https://doi.org/10.1103/PhysRevA.89.032509

[44] S. Willitsch, 'Experimental Methods in Cation Spectroscopy' in 'Handbook of High-Resolution Spectroscopy’, Ed. M. Quack, F. Merkt, John Wiley \& Sons, 2011.

[45] X. Tong, A. H. Winney, S. Willitsch, Phys. Rev. Lett. 2010, 105, 143001 https://doi.org/10.1103/PhysRevLett.105.143001

[46] Y.-D. Wu, et al., Chin. J. Chem. Phys. 2007, 20, 285 , https://doi.org/10.1088/1674-0068/20/03/285-290

[47] I. H. Bachir, H. Bolvin, C. Demuynck, J. Destombes, A. Zellagui, J. Mol. Spectrosc. 1994, 166, 88, https://doi.org/10.1006/jmsp.1994.1174

[48] K. Harada, T. Wada, T. Tanaka, J. Mol. Spectrosc. 1994, 163, 436, https://doi.org/10.1006/jmsp.1994.1038

[49] D. C. Cartwright, J. Chem. Phys. 1973, 58, 178, https://doi.org/10.1063/1.1678902

[50] S. R. Langhoff, C. W. Bauschlicher Jr., H. Partridge, J. Chem. Phys. 1987, 87, 4716, https://doi.org/10.1063/1.452835

[51] F. R. Gilmore, R. R. Laher, P. J. Espy, J. Phys. Chem. Ref. Data 1992, 21, 1005, https://doi.org/10.1063/1.555910

\section{License and Terms}

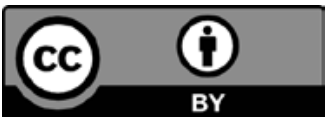

This is an Open Access article under the terms of the Creative Commons Attribution License CC BY 4.0. The material may not be used for commercial purposes.

The license is subject to the CHIMIA terms and conditions: (http:// chimia.ch/component/sppagebuilder/?view=page \&id=12).

The definitive version of this article is the electronic one that can be found at https://doi.org/10.2533/chimia.2021.291 